\title{
Phylogenetic analysis in a recent controlled outbreak of Crimean-Congo haemorrhagic fever in the south of Iran, December 2008
}

\author{
S Chinikar (chinikar@pasteur.ac.ir) ${ }^{1}$, S M Ghiasi ${ }^{1}$, M Moradi ${ }^{1}$, M M Goya ${ }^{2}$, M Reza Shirzadi ${ }^{2}$, M Zeinali ${ }^{2}$, E Mostafavi ${ }^{1}$, \\ M Pourahmad³, A Haeri3,4 \\ 1. National Reference Laboratory for Arboviruses and Viral Haemorrhagic Fevers, Pasteur Institute of Iran, Tehran, Iran \\ 2. Centre for Disease Control (CDC), Ministry of Health of Iran, Tehran, Iran \\ 3. Jahrom Faculty of Medicine \\ 4. Shahid Beheshti University of Medical Sciences, Tehran, Iran
}

Citation style for this article:

Chinikar S, Mojtaba Ghiasi S, Moradi M, Goya MM, Reza Shirzadi M, Zeinali M, Mostafavi E, Pourahmad M, Haeri A. Phylogenetic analysis in a recent controlled outbreak of Crimean-Congo haemorrhagic fever in the south of Iran, December 2008. Euro Surveill. 2010;15(47):pii=19720. Available online: http://www.

eurosurveillance.org/ViewArticle.aspx?Articleld=19720

Article published on 25 November 2010

Crimean-Congo haemorrhagic fever (CCHF) is a viral zoonotic disease with a high mortality rate in humans. The CCHF virus is transmitted to humans through the bite of Ixodid ticks or contact with blood or tissues of CCHF patients or infected livestock. In December

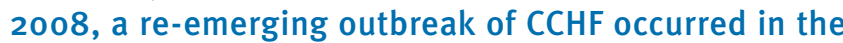
southern part of Iran. Five people were hospitalised with sudden fever and haemorrhaging, and CCHF was confirmed by RT-PCR and serological assays. One of the cases had a fulminant course and died. Livestock was identified as the source of infection; all animals in the incriminated herd were serologically analysed and more than half of them were positive for CCHFV. We demonstrated that two routes of transmission played a role in this outbreak: contact with tissue and blood of infected livestock, and nosocomial transmission. Phylogenetic analyses helped to identify the origin of this transmission. This outbreak should be considered as a warning for the national CCHF surveillance system to avoid further outbreaks through robust prevention and control programmes.

\section{Introduction}

Crimean-Congo haemorrhagic fever (CCHF) is a viral zoonotic haemorrhagic fever with up to $13-50 \%$ mortality rate in humans. Infected animals are unsymptomatic. The disease is caused by Crimean-Congo haemorrhagic fever virus (CCHFV) that belongs to the family Bunyaviridae, genus Nairovirus. The of negative single-stranded RNA genome consists of three segments, large (L), medium (M) and small (S), coding for the viral polymerase (L), the envelope glycoproteins (M) and the viral nucleoprotein (S) [1-5]. The typical course of CCHF progresses through four distinct phases: incubation, pre-haemorrhagic phase, haemorrhagic phase and convalescence [6-8]. After a incubation period of one to three days, the patient has sudden onset of fever, myalgia, nausea and severe headache. Within three to six days of the onset of illness, a petechial rash and haemorrhagic symptoms such as epistaxis, haematemesis, and melaena may occur. The most severely ill patients develop multiorgan failure characterised by shock, haemorrhaging and coma [9-11]. The virus is transmitted to humans through the bite of Ixodid ticks or by contact with blood or tissues from infected livestock [12-14]. In addition to zoonotic transmission, CCHFV can be spread from person to person and is one of the rare haemorrhagic fever viruses able to cause nosocomial outbreaks in hospitals [15-20].

In the period from 1 January 2000 to 12 September 2010, 738 confirmed cases of CCHF and 108 associated fatalities were notified in Iran $[15,21]$. The province reporting most infections was Sistan-va-Baluchistan, Isfahan and Fars (Figure 1).

\section{FIGURE 1}

Geographical distribution of Crimean-Congo haemorrhagic fever in Iran, 1 January 2000-12 September $2010(n=738)$

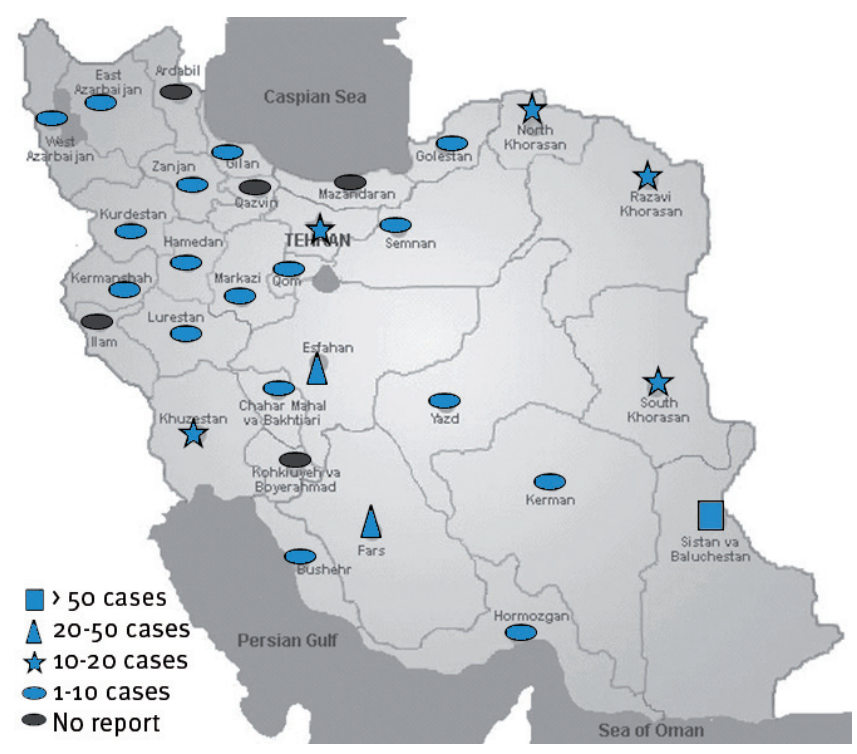




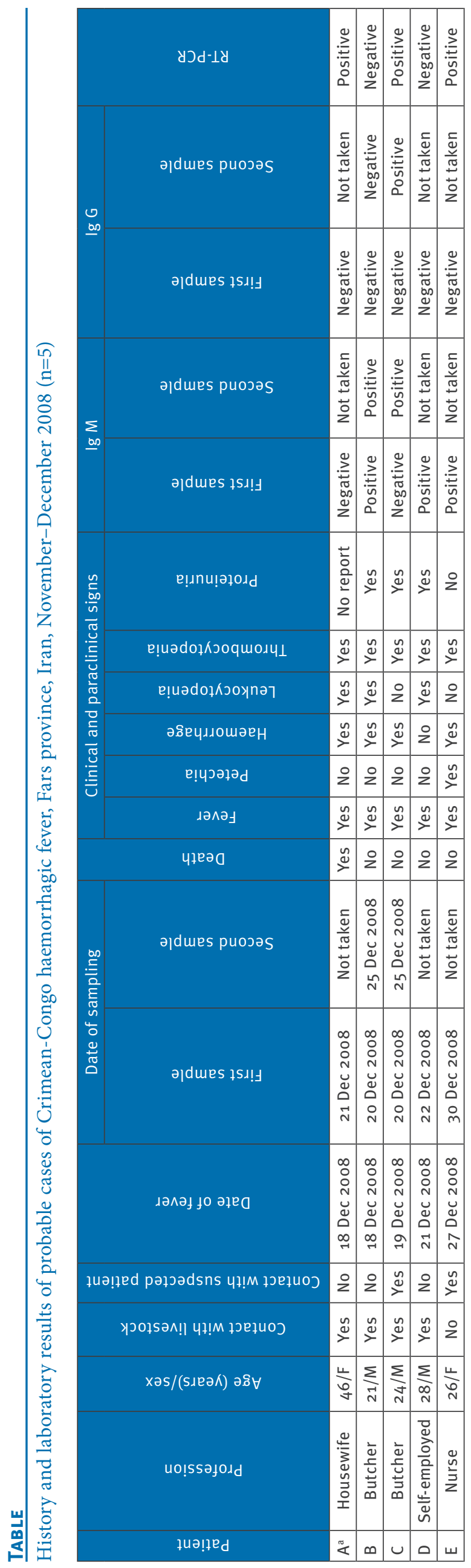

Outbreak description

Here, we report a CCHF outbreak in Fars province, Iran, caused by contact of humans with blood or tissues of infected livestock, with additional nosocomial transmission. In total, five patients (A-E) were admitted to the regional hospital with similar presentations of a haemorrhagic condition in the period from 18 December to 21 December 2008. This period coincides with the Muslim ceremony Eid-al-Adha (the ceremony of sacrificing livestock) which is celebrated in Islamic countries on 9 December.

Patients A and D (who are part of the same family) bought a calf from a butchery run by two brothers, patients $B$ and $C$, and hired them to sacrifice the animal. On the morning of 18 December 2008, Patient A, the index case of this outbreak, was admitted to hospital and died after a fulminant course of CCHF. In the evening of the same day, Patients B and C were admitted to the same hospital with fever and chill, severe headache, dizziness, photophobia. Patient $\mathrm{D}$ developed similar clinical signs on 21 December and was hospitalised. Nine days after the index case, the nurse caring of these four patients was also hospitalised with haemorrhagic symptoms (Patient E).

\section{Materials and methods \\ Case definition}

The case definition for probable cases included patients admitted between 18 December and 27 December 2008 in the regional hospital and presenting with a clinical picture compatible with CCHF, or contact with tissues or blood from a possibly infected animal, or a healthcare worker with a history of contact with a CCHF case. Probable cases with positive IgM serology and/or positive RT-PCR were considered as CCHF confirmed cases.

\section{Laboratory analysis}

Human and animal sera were analysed by ELISA for anti-CCHFV IgM and IgG as described [15,22]. Viral RNA was extracted from patient's serum using QIAamp RNA Mini kit (QIAgen GmbH, Hilden, Germany) and analysed by gel-based and real-time RT-PCR with a one-step RT-PCR kit (QIAgen GmbH, Hilden, Germany). A 536 bp fragment of the $S$ segment of the CCHFV genome was amplified $[4,8,12]$ and sequenced.

Phylogenetic analysis was performed with the neighbour-joining method based on Kimura two-parameter distances by using Mega 4 software. Bootstrap confidence limits were based on 500 replicates. Evolutionary divergence, distance matrix and subsequently sets of phylogenetic trees were calculated by the software [23].

\section{Results and discussion}

As summarised in the Table, five probable CCHF cases in this outbreak were confirmed by serological and molecular methods. It is worth mentioning that no immunological response was detected in the fatal case that had a fulminant course, Patient A, and CCHF 
in this case was only confirmed by a strongly positive RT-PCR. There is evidence of other fatal cases lacking an immune response to CCHFV $[6,24]$. Patients $C$ and $E$ were positive both for viral RNA and antibodies against CCHFV, while Patients $B$ and $D$ were negative in the PCR and only confirmed by serological assay. Notably, ribavirin was administered to the patients in hospital.

At the same time, serum samples were collected from 50 animals in the herd from which the calf had been bought. In 30 of these samples antibodies to CCHFV were detected. Although CCHF is an asymptomatic disease in livestock that does not kill the animals, seroepidemiological surveys of animal populations in endemic areas and high risk regions could be useful in that they may complement the national surveillance system and serve as an early warning of CCHF in the area.

In this outbreak, it was demonstrated that the main transmission route of CCHF was through handling blood or tissues of infected livestock (for patients $A, B, C$ and D), while patient $E$ had had no contact to livestock and was infected nosocomially. It is unclear why Patient $A$ had such a fulminant course of disease and died. Patients B, C and D were infected through the same route, by direct contact with tissue and blood of the same animal, but had a milder course of disease and recovered. It is important in infectious disease outbreaks to investigate what factors determine the severity of the disease in different individuals [6]. There are published reports on the influence of cytokine levels on the immune response to CCHFV in different patients $[24,25]$. It has been shown that patients infected with a higher dose of virus develop more severe disease symptoms and outcomes [26-28]. Although we did not use quantitative RT-PCR, the band density of the PCR product obtained from patient $A$ was much higher than that of the other patients. On the other hand, it seems likely that patients $B$ and $C$ presented a mild form of the disease because they may already have had antibodies against CCHFV due to their professional exposure. Moreover, no anti-CCHFV IgG antibody response was detected for the patient $B$, whereas a normal serological and molecular pattern was seen in patient $C$, which

\section{FIGURE 2}

Phylogenetic comparison of Crimean-Congo haemorrhagic fever virus isolates from Iran with isolates form patients in the recent outbreak in Fars province, Iran, December 2008

A

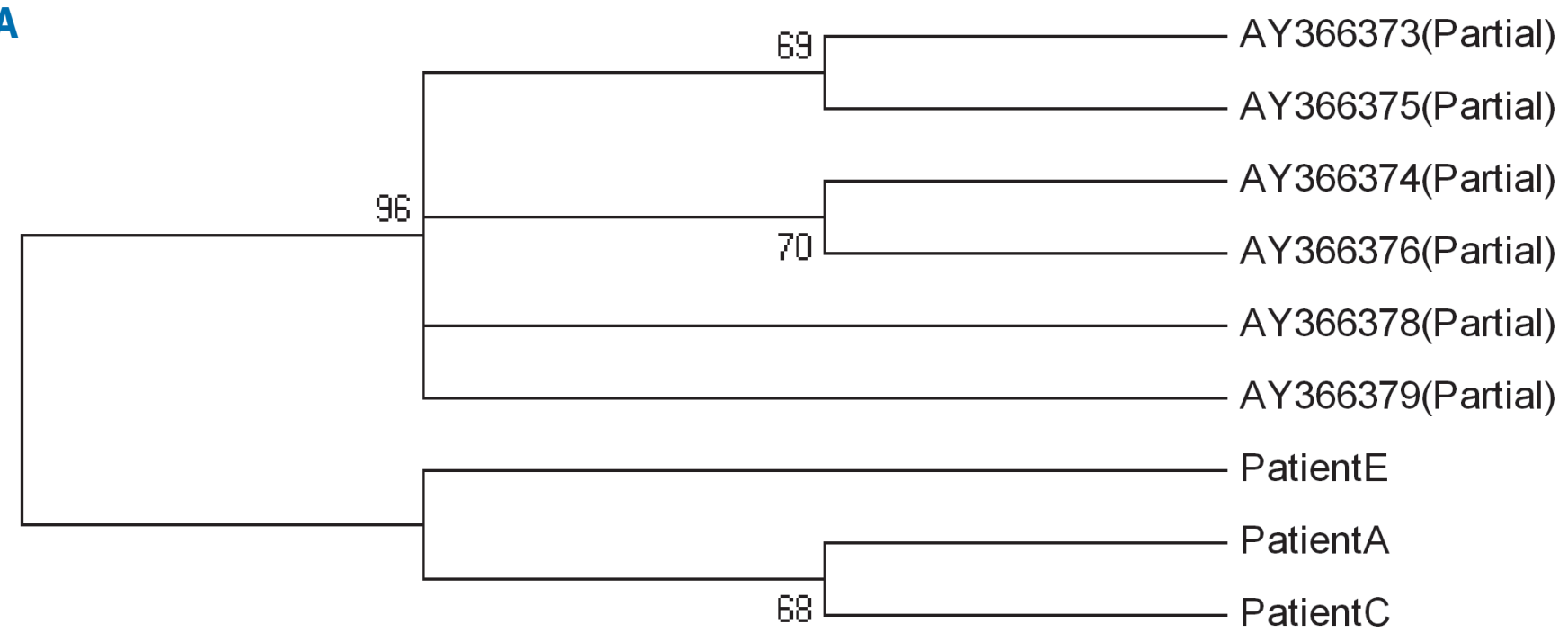

B

\begin{tabular}{|c|c|c|c|c|c|c|c|c|c|c|c|c|}
\hline & \multicolumn{11}{|c|}{ Percent identity } & \\
\hline \multirow{11}{*}{ 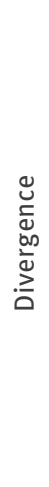 } & & 1 & 2 & 3 & 4 & 5 & 6 & 7 & 8 & 9 & & \\
\hline & 1 & & 99.9 & 99.9 & 99.9 & 99.9 & 99.8 & 97.6 & 97.6 & 97.7 & 1 & $\mathrm{AY}_{366373 \text { (partial) }}$ \\
\hline & 2 & 0.2 & & 100.0 & 99.9 & 99.9 & 99.9 & 97.6 & 97.6 & 97.7 & 2 & $\mathrm{AY}_{366379 \text { (partial) }}$ \\
\hline & 3 & 0.2 & 0.0 & & 99.9 & 99.9 & 99.9 & 97.6 & 97.6 & 97.7 & 3 & $\mathrm{AY}_{366378 \text { (partial) }}$ \\
\hline & 4 & 0.2 & 0.4 & 0.4 & & 99.8 & 99.8 & 97.5 & 97.6 & 97.6 & 4 & $\mathrm{AY}_{366375 \text { (partial) }}$ \\
\hline & 5 & 0.4 & 0.2 & 0.2 & 0.6 & & 99.9 & 97.6 & 97.6 & 97.7 & 5 & $\mathrm{AY}_{366374 \text { (partial) }}$ \\
\hline & 6 & 0.6 & 0.4 & 0.4 & 0.8 & 0.2 & & 97.5 & 97.5 & 97.6 & 6 & $\mathrm{AY}_{366376 \text { (partial) }}$ \\
\hline & 7 & 1.0 & 0.8 & 0.8 & 1.2 & 1.0 & 1.2 & & 99.9 & 99.8 & 7 & Patient A \\
\hline & 8 & 1.0 & 0.8 & 0.8 & 1.3 & 1.0 & 1.3 & 0.2 & & 99.9 & 8 & Patient C \\
\hline & 9 & 0.8 & 0.6 & 0.6 & 1.0 & 0.8 & 1.0 & 0.4 & 0.2 & & 9 & Patient E \\
\hline & & 1 & 2 & 3 & 4 & 5 & 6 & 7 & 8 & 9 & & \\
\hline
\end{tabular}

A. The phylogeny tree of nucleotide sequences spanning described regions of the S-segment of CCHF virus genome which is detected in the outbreak. B. Nucleotide identity and divergence of CCHF virus genomes isolated from patients of the outbreak. 
might be interpreted to indicate that patient B was infected with a very low viral dose. Recent investigations have concluded a relationship linking the severity and outcome of CCHFV infections with the strength of the host immune response and the initial viral load $[24,25,27]$.

Phylogenetic analysis of alignments of three partial genomic sequences ( $536 \mathrm{bp}$ ) of the CCHFV S segment indicates that the viruses isolated from patients $A, C$ and $E$ can be differentiated into two distinct branches, with a slightly lower identity between patients $A$ and E. As illustrated in Figure 2, the sequences obtained in this outbreak are not clustered with other CCHFV sequences isolated in Iran (about 97.5\% identity). It is possible that a new strain occurred in the outbreak region, and further phylogenetic analyses are required to identify the precise origin of this genetic variant. However, comparison of the isolates from our patients with isolates from other areas may give some indications as to the origin of this outbreak [12].

One of the factors that contributed to the control of this outbreak was the well-coordinated and efficient surveillance system for CCHF and other viral haemorrhagic fevers that is in place in Iran. The system is not only responsible for continuous monitoring of these diseases but also deals with outbreaks. Rapid and precise laboratory diagnosis of CCHF allowed controlling this outbreak. Nevertheless, a higher level of training and precautionary measures for healthcare workers (such as use of isolation chambers in hospital wards, mask and other medical shields during contact to CCHF patients) and other high risk professions could help to decrease the outbreak rate in the endemic areas. In conclusion, with Iran being an endemic country for CCHF in the Middle East and neighbouring Turkey an endemic country in Europe, efficient surveillance and control programmes on CCHF in Iran could prove beneficial also for the European region.

\section{Acknowledgements}

We would like to thank the other members of the National Reference Laboratory for Arboviruses and Viral Haemorrhagic Fevers at Pasteur Institute of Iran for their technical support. This study was performed funded by the budget of the National Reference Laboratory for Arboviruses and Viral Haemorrhagic Fevers at Pasteur Institute of Iran.

*Authors' correction: At the request of the authors, the name of the author Seyed Mojtaba Ghiasi was corrected on 12 April 2010.

\section{References}

1. Donets MA, Chumakov MP, Korolev MB, Rubin SG. Physicochemical characteristics, morphology and morphogenesis of virions of the causative agent of Crimean hemorrhagic fever. Intervirology. 1977;8(5):294-308.

2. Marriott AC, Nuttall PA. Comparison of the $S$ RNA segments and nucleoprotein sequences of Crimean-Congo hemorrhagic fever, Hazara and Dugbe viruses. Virology. 1992;189(2):795-9.

3. Martin ML, Lindsey-Regnery H, Sasso DR, McCormick JB, Palmer E. Distinction between Bunyaviridae genera by surface structure and comparison with Hantaan Virus using negative stain electron microscopy. Arch Virol. 1985;86(1-2):17-28.
4. Papa A, Bozovi B, Pavlidou V, Papadimitriou E, Pelemis M, Antoniadis A. Genetic detection and Isolation of CrimeanCongo hemorrhagic fever virus Kosovo, Yugoslavia. Emerg Infect Dis. 2002;8(8):852-4.

5. Sanchez AJ, Vincent MJ, Nichol ST. Characterization of the glycoproteins of Crimean-Congo hemorrhagic fever virus. Virol. 2002;76(14):7263-75.

6. Ergonul O, Celikbas A, Dokuzoguz B, Eren S, Baykam N, Esener $\mathrm{H}$. Characteristics of patients with Crimean-Congo hemorrhagic fever in a recent outbreak in Turkey and impact of oral ribavirin therapy. Clin Infect Dis. 2004;39(2):284-7.

7. Ergonul O, Whitehouse CA. Crimean-Congo Hemorrhagic Fever. A Global Perspective. The Netherlands:Springer. 2007: 250-470.

8. Whitehouse CA. Crimean-Congo hemorrhagic fever. Antiviral Res. 2004;64(3):145-60.

9. Paragas J, Whitehouse CA, Endy TP, Bray M. A simple assay for determining antiviral activity against Crimean-Congo hemorrhagic fever virus. Antiviral Res. 2004;62(1):21-5.

10. Swanepoel R, Gill DE, Shepherd AJ, Leman PA, Mynhardt $\mathrm{JH}$, Harvey S. The clinical pathology of Crimean-Congo hemorrhagic fever. Rev Infect Dis. 1989;11 Suppl 4:S794-800.

11. Swanepoel R. Nairovirus infections. In: Porterfield JS, editor. Exotic viral infections. London: Chapman and Hall; 1995;285-93.

12. Chinikar S, Persson SM, Johansson M, Bladh L, Goya M, Houshmand B, et al. Genetic analysis of Crimean-Congo hemorrhagic fever virus in Iran. J Med Virol. 2004;73(3): 404-11.

13. Logan TM, Linthicum KJ, Bailey CL, Watts DM, Moulton JR. Experimental transmission of Crimean-Congo hemorrhagic fever virus by Hyalomma truncatum Koch. Am J Trop Med Hyg. 1989;40(2):207-12.

14. Shepherd AJ, Swanepoel R, Shepherd SP, Leman PA, Mathee 0 . Viremic transmission of Crimean-Congo hemorrhagic fever virus to ticks. Epidemiol Infect. 1991;106(2):373-82.

15. Chinikar S, Ghiasi SM, Hewson R, Moradi M, Haeri A. CrimeanCongo hemorrhagic fever in Iran and neighboring countries. J Clin Virol. 2010;47(2):110-14.

16. Fisher-Hoch SP, Khan JA, Rehman S, Mirza S, Khurshid M, McCormick JB. Crimean-Congo hemorrhagic fever treated with oral ribavirin. Lancet. 1995; 346(8973):472-5.

17. Gonzalez JP, Camicas JL, Cornet JP, Faye O, Wilson ML. Sexual and transovarian transmission of Crimean-Congo hemorrhagic fever virus in Hyalomma truncatum ticks. Res Virol. 1992;143(1):23-8.

18. Hoogstraal H. The epidemiology of tick-born Crimean-Congo hemorrhagic fever in Asia, Europe, and Africa. J Med Entomol. 1979;15(4):307-417.

19. Van Eeden PJ, Joubert JR, Van de Wal BW, King JB, de Kock A, Groenewald JH. A nosocomial outbreak of Crimean-Congo haemorrhagic fever at Tygerberg Hospital. Part 1. Clinical Features. S Afr Med J. 1985;68(10):711-7.

20. Vorou R, Pierroutsakos IN, Maltezou HC. Crimean-Congo hemorrhagic fever. Curr Opin Infect Dis. 2007;20(5):495-500.

21. Chinikar S, Goya MM, Shirzadi MR, Ghiasi SM, Mirahmadi R, Haeri A, et al. Surveillance and Laboratory Detection System of Crimean-Congo Hemorrhagic Fever in Iran. Transbound Emerg Dis. 2008;55(5-6):200-4.

22. Garcia S, Chinikar S, Coudrier D, Billecocq A, Hooshmand B, Crance JM, et al. Evaluation of a Crimean-Congo hemorrhagic fever virus recombinant antigen expressed by Semliki Forest Suicide virus for IgM and IgG antibody detection in human and animal sera collected in Iran. J Clin Virol. 2006;35(2):154-9.

23. Tamura K, Dudley J, Nei M, Kumar S. MEGA4: Molecular Evolutionary Genetics Analysis (MEGA) software version 4.0. Mol Biol Evol. 2007;24(8):1596-9.

24. Papa A, Bino S, Velo E, Harxhi A, Kota M, Antoniadis A. Cytokine levels in Crimean-Congo hemorrhagic fever. J Clin Virol. 2006;36(4):272-6.

25. Weber F, Mirazimi A. Interferon and cytokine responses to Crimean-Congo hemorrhagic fever virus; an emerging and neglected viral zoonosis. Cytokine Growth Factor Rev. 2008;19(5-6):395-404.

26. Duh D, Saksida A, Petrovec M, Ahmeti S, Dedushaj I, Panning $M$, et al. Viral load as predictor of Crimean-Congo hemorrhagic fever outcome. Emerg Infect Dis. 2007;13(11):1769-72.

27. Papa A, Drosten C, Bino S, Papadimitriou E, Panning M, Velo E, et al. Viral load and Crimean-Congo hemorrhagic fever. Emerg Infect Dis. 2007;13(5): 805-6.

28. Wölfel R, Paweska JT, Petersen N, Grobbelaar AA, Leman PA, Hewson R, et al. Virus detection and monitoring of viral load in Crimean-Congo hemorrhagic fever virus patients. Emerg Infect Dis. $2007 ; 13(7): 1097-100$. 